\title{
Epigenetic Robotics: Behavioral Treatments and Potential New Models for Developmental Pediatrics
}

$\mathrm{T}$ The field of epigenetic or developmental robotics is a new interdisciplinary area at the intersection of child development and robotics $(1,2)$. The goals of this area include the use of robots in therapy with children, the use of robots in modeling the typical and atypical development of children, and creating better robots. Epigenetic robotics stands poised to make strong theoretical contributions to our understanding of child development. A core area of research in epigenetic robotics is the study of development over longtime periods, or what is called openended development (3), ongoing emergence (4), or autonomous mental development (5). By offering insights into the mechanisms of developmental processes, epigenetic robotics may contribute in fundamentally interdisciplinary ways to our understanding of child development. The purpose of this brief review is to illustrate what epigenetic robotics has to offer the field of pediatrics, and also to consider what pediatrics might offer epigenetic robotics. We consider two areas where epigenetic robotics can contribute to developmental pediatrics: the use of robots in therapy, and in modeling the typical and atypical development of children.

\section{ROBOTS IN THERAPY}

An application area of epigenetic robotics is the use of robots in therapy with children, where those children have cognitive deficits such as autism $(6,7)$. The advantages of using robots in some forms of therapy with these children include possibly less threatening social interactions relative to humans, and control over the interaction complexity between the child and the

\author{
Christopher G. Prince \\ Lakshmi J. Gogate
}

social partner (6). One strategy taken in this research is to use relatively simple robots. For example, one study explored "if a mobile robot could, by being ... predictable, attractive and simple, facilitate reciprocal interaction such as imitation" (8). This study found some improvement of shared attention when autistic children interacted with robots as opposed to interacting with a human. Another group of researchers have also used a relatively simple robot in interactions with children with autism spectrum and other disorders (9). These researchers have followed individual children's interaction with the robots over extended time periods (e.g., for as long as $2 \frac{1}{2}$ years). Figure 1 depicts the robot used in this study. These studies used partly-autonomous tele-operated robots $(8,9)$. Robotic techniques also make it possible to create robot-perspective video and audio records of the interactions with the children. These records can be useful for therapists when developing treatment plans.

A different strategy in the investigation of robots as therapeutic agents with children is to use robots that are more similar to humans in their form. While the physical complexity of the robot will then be similar to a human, behavioral complexity (e.g., the robot's predictability) can still be simplified relative to a human. For example, one project $(10,11)$ used a humanoid robot that was similar to a human head and was capable of expressing and modulating facial expressions depicting six basic emotions (e.g., happiness, sadness). These researchers have also used this robot in autism therapy. Preliminary findings suggest that an autistic child did not find the robot threatening or surprising (10), and that autistic children socially interact with the robot by imitating and drawing another person's attention to the robot (11).

\section{ROBOTS AS MODELS OF TYPICAL AND ATYPICAL DEVELOPMENT OF CHILDREN}

Epigenetic robotics also involves the creation of models related to various developmental phenomena. By incorporating sensors and/or motors, i.e., by having a body, epigenetic robots draw on the hypothesis that bodies and sensorimotor interaction with the world influence development (12). For example, it has been hypothesized that young infants' limited visual acuity can actually serve to help them learn (13). Computational models with visual sensory inputs have been useful in illustrating the validity of this hypothesis $(14,15)$. Similarly, it has been hypothesized that infants can learn aspects of motor control by gradually releasing the number of motor degrees of freedom they use over time (16). Robots interacting with physical environments have been used to test this hypothesis $(17,18,19)$.

Robotic models of the development of social and language skills are also being forwarded. For example, one project (20) has implemented aspects of a Theory of Mind Mechanism (21) in a robotic system. Briefly, the robot ("Cog") was an upper-torso humanoid robot (including arms, trunk, head, \& neck) and had a visual attention system, detected eyes and faces, and discriminated animate from inanimate motions (22). Robotic systems can also 


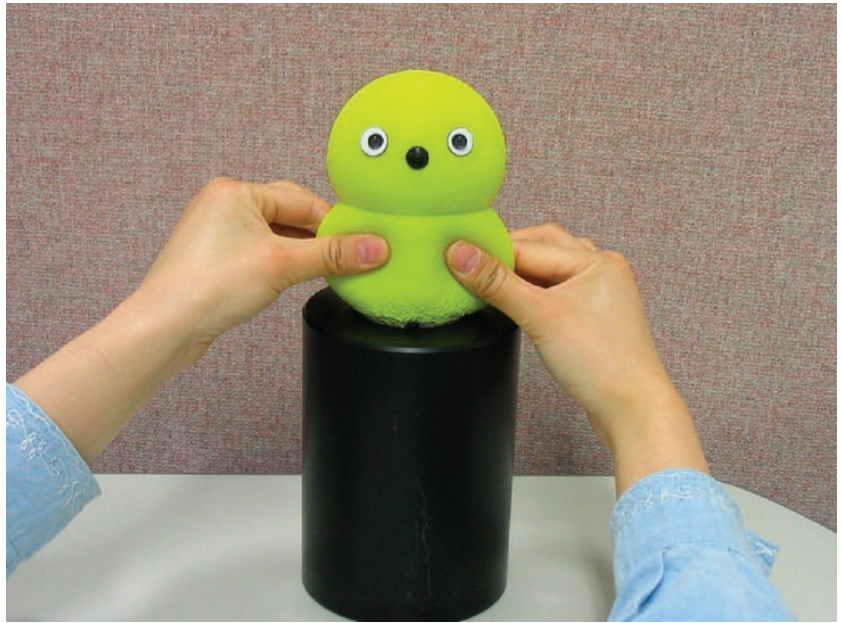

Figure 1. "Keepon" Robot Used in Autism Therapy with Children. Reprinted from Kozima K and Nakagawa C, Interactive robots as facilitators of children's social development. In: Kordic V, Lazinica A, Merden M (Eds) Mobile Robotics: Toward New Applications, Advanced Robotics Systems, with permission from the authors.

exhibit aspects of joint attention, where a child and a caregiver share attention regarding an object. For example, one robot learned to use the face and eye-gaze direction of a human to appropriately turn to look at an interesting object (23). Still other systems are being programmed to learn to imitate facial gestures (24), and to learn to map words to objects and actions (25).

It is also possible to model atypical development using epigenetic robotics. Unlike animal models we have no ethical prohibitions against altering either the internal components or external environments of a robot. For example, if a robot has several sensory modalities (e.g., vision, audition, and touch), one of those modalities can be eliminated or disabled to model sensory disability and subsequent development. This area is ripe for new research in epigenetic robotics. Various basic skills have been demonstrated in robots, e.g., the use of visual and acoustic cues (26) and tactile sensation (27), and researchers are just starting to apply these basic skills to systems that might model atypical development.

Some progress has been made in models of atypical development, toward a robotic model of attentiondeficit hyperactivity disorder (ADHD) (28). In our own research, we have started using sensory-oriented stimuli analysis techniques in studies of auditory-visual perception of full-term and preterm infants. For example, infant experiments show that relative to fullterms, preterm infants have an attenuated ability to detect synchrony between an adult's spoken utterances and hand-held object motions (29). Using the same stimuli as with the preterms, we have shown that full-term infants' performance generally matches that of a computational model of audio-visual synchrony detection (unpublished data). Before closing, it is important to note that non-robotic computational models of aspects of typical and atypical child development have been forwarded (30). For example, a nonrobotic model has simulated the gaze following of children with autism and Williams Syndrome (31). Robotic models, in contrast to non-robotic models, have particular strength where sensorimotor issues and/or physical world interactions are vital to the hypothesis being studied.

\section{CONCLUSION}

In this paper, we have briefly reviewed two areas where epigenetic robotics can contribute to developmental pediatrics: The use of robots in therapeutic settings and the use of robots as models of typical or atypical development. Robots can be useful in therapy with children because they can provide less threatening social interactions, and offer more control over the interaction complexity. We believe that pediatrics also stands poised to make a substantial contribution to epigenetic robotics. Researchers in epigenetic robotics are interested in how robots can be used in a variety of clinical contexts as both models and therapeutic agents. Pediatrics offers a wealth of real world clinical problems upon which to apply both the models and the therapeutic agents of epigenetic robotics. We encourage interested pediatricians and researchers to make contact with the epigenetic robotics community, and to attend conferences in this area (e.g., see http:// www.epigenetic-robotics.org; http:// www.mentaldev.org).

\section{REFERENCES}

1. Asada M, MacDorman K, Ishiguro H, Kuniyoshi Y 2001 Cognitive developmental robotics as a new paradigm for the design of humanoid robots. Robot Auton Syst 37:185-193

2. Lungarella M, Metta G, Pfeifer R, Sandini G 2003 Developmental robotics: A survey. Connect Sci 15:151-190

3. Oudeyer P-Y, Kaplan F 2006 Discovering communication. Connect Sci 18:189-206

4. Prince CG, Helder NA, Hollich GJ 2005 Ongoing emergence: A core concept in epigenetic robotics. In: Berthouze L, Kaplan F, Kozima H, Yano Y, Konczak J, Metta G, Nadel J, Sandini G, Stojanov G, Balkenius C (Eds) Proceedings of The Fifth International Workshop on Epigenetic Robotics: Modeling Cognitive Development in Robotic Systems. Lund University Cognitive Studies, Lund, pp 63-70

5. Weng J, McClelland J, Pentland A, Sporns O, Stockman I, Sur M, Thelen E 2001 Autonomous mental development by robots and animals. Science 291:599-600

6. Dautenhahn K, Werry I, Salter T, te Boekhorst R 2003 Towards adaptive autonomous robots in autism therapy: Varieties of interactions. In: Proceedings of the IEEE International Symposium on Computational Intelligence in Robotics and Automation, pp 577-582

7. Scassellati B 2005 Using robots to study abnormal social development. In: Berthouze L, Kaplan F, Kozima H, Yano Y, Konczak J, Metta G, Nadel J, Sandini G, Stojanov G, Balkenius C (Eds) Proceedings of The Fifth International Workshop on Epigenetic Robotics: Modeling Cognitive Development in Robotic Systems. Lund University Cognitive Studies, Lund, pp 11-14

8. Duquette A, Mercier H, Michaud F 2006. Investigating the use of a mobile robotic toy as an imitation agent for children with autism. In: Kaplan F, Oudeyer P-Y, Revel A, Gaussier P, Nadel J, Berthouze L, Kozima H, Prince CG, Balkenius C (Eds) Proceedings of the Sixth International Workshop on Epigenetic Robotics: Modeling Cognitive Development in Robotic Systems. Lund University Cognitive Studies, Lund, pp 167-168

9. Kozima H, Nakagawa $C$ (in press) Interactive robots as facilitators of children's social development. In: Kordic V, Lazinica A, Merden M (Eds) Mobile Robotics: Towards New Applications, Advanced Robotics Systems

10. Pioggia G, Igliozzi R, Ferro M, Ahluwalia A, Muratori F, De Rossi D 2005 An android for enhancing social skills and emotion recognition in people with autism. IEEE Trans Neural Syst Rehabil Eng 13:507-515

11. Pioggia G, Ferro M, Sica ML, Dalle Mura G, Casalini S, De Rossi D, Igliozzi R, Muratori F 2006 Imitation and learning of the emotional behavior: 
Towards an android treatment for people with autism. In: Kaplan F, Oudeyer P-Y, Revel A, Gaussier P, Nadel J, Berthouze L, Kozima H, Prince CG, Balkenius C (Eds) Proceedings of the Sixth International Workshop on Epigenetic Robotics: Modeling Cognitive Development in Robotic Systems. Lund University Cognitive Studies, Lund, pp 119-125

12. Pfeifer R, Bongard J 2006 How the Body Shapes the Way We Think: A New View of Intelligence. MIT Press, Cambridge, MA

13. Turkewitz G, Kenny P 1982 Limitations on input as a basis for neural organization and perceptual development: a preliminary theoretical statement. Dev Psychobiol 15:357-368

14. Dominguez M, Jacobs RA 2003 Developmenta constraints aid the acquisition of binocular disparity sensitivities. Neural Comput 15:161-182

15. Jacobs RA, Dominguez M 2003 Visual development and the acquisition of motion velocity sensitivities. Neural Comput 15:761-781

16. Thelen E, Schöner G, Scheier C, Smith LB 2001 The dynamics of embodiment: A field theory of infant perseverative reaching. Behav Brain Sci 24:1-86

17. Berthouze L, Lungarella M 2004 Motor skill acquisition under environmental perturbations: on the necessity of alternate freezing and freeing of degrees of freedom. Adapt Behav 12:47-64

18. Lungarella M, Berthouze L 2002 On the interplay between morphological, neural and environmental dynamics: A robotic case-study. Adapt Behav 10:223-241

19. Veskos P, Demiris Y 2005 Developmental acquisition of entrainment skills in robot swinging using van der Pol oscillators. In: Berthouze L, Kaplan F, Kozima H, Yano Y, Konczak J, Metta G, Nadel J,
Sandini G, Stojanov G, Balkenius C (Eds) Proceedings of The Fifth International Workshop on Epigenetic Robotics: Modeling Cognitive Development in Robotic Systems. Lund University Cognitive Studies, Lund, pp 87-93

20. Scassellati B 2002 Theory of mind for a humanoid robot. Autonomous Robots 12:13-24

21. Baron-Cohen S 1995 Mindblindness. MIT Press, Cambridge, MA

22. Scassellati B 2001 Discriminating animate from inanimate visual stimuli. In: Proceedings of the Seventeenth International Joint Conference on Artificial Intelligence, pp 1405-1410

23. Nagai Y, Hosoda K, Morita A, Asada M 2003 A constructive model for the development of joint attention. Connection Science 15:211-229

24. Breazeal C, Buchsbaum D, Gray J, Gatenby D, Blumberg B 2005 Learning from and about others: Towards using imitation to bootstrap the social understanding of others by robots. Artif Life 11:31-62

25. Yu C, Ballard DH, Aslin RN 2005 The role of embodied intention in early lexical acquisition. Cognit Sci 29:961-1005

26. Natale L, Metta G, Sandini G 2002 Development of auditory-evoked reflexes: visuo-acoustic cues integration in a binocular head. Robot Autonom Syst 39:87-106

27. Natale L, Torres-Jara E 2006 A sensitive approach to grasping. In: Kaplan F, Oudeyer P-Y, Revel A, Gaussier P, Nadel J, Berthouze L, Kozima H, Prince CG, Balkenius C (Eds) Proceedings of the Sixth International Workshop on Epigenetic Robotics: Modeling Cognitive Development in Robotic Systems. Lund University Cognitive Studies, Lund, pp 87-94
28. Balkenius C, Björne P 2001 Toward a robot model of attention-deficit hyperactivity disorder (ADHD). In: Balkenius C, Zlatev J, Kozima H, Dautenhahn K, Breazeal C (Eds) Proceedings of the First International Workshop on Epigenetic Robotics: Modeling Cognitive Development in Robotic Systems. Lund University Cognitive Studies, Lund, pp 61-67

29. Gogate LJ, Awal D, Prince CG 2006 Preterm and full-term infants' invariance detection and vocabulary development: A longitudinal study. In: Gogate J (Chair) Invariance detection and speechlanguage development: What is the mechanism? Symposium Presented at the International Conference on Infant Studies, Kyoto, Japan

30. Quinlan PT (Ed) 2003 Connectionist Models of Development: Developmental Processes in Real and Artificial Neural Networks. Psychology Press, New York

31. Triesch J, Teuscher C, Deák GO, Carlson E 2006 Gaze following: why (not) learn it? Dev Sci 9:125157

Christopher G. Prince

Department of Computer Science

University of Minnesota Duluth

Duluth, MN 55812

Lakshmi J. Gogate

Department of Pediatrics and Psychiatry

SUNY Health Science Center at Brooklyn

Brooklyn, NY 11203

DOI: $10.1203 /$ pdr.0b013e3180459fdd 